San Jose State University

SJSU ScholarWorks

Master's Theses

Master's Theses and Graduate Research

1991

\title{
A retrospective study to determine the relationship between selected hemocomponents, ultrafiltration, and body temperature
}

Nancy A. Smith

San Jose State University

Follow this and additional works at: https://scholarworks.sjsu.edu/etd_theses

\section{Recommended Citation}

Smith, Nancy A., "A retrospective study to determine the relationship between selected hemocomponents, ultrafiltration, and body temperature" (1991). Master's Theses. 216.

DOI: https://doi.org/10.31979/etd.de3y-e95q

https://scholarworks.sjsu.edu/etd_theses/216

This Thesis is brought to you for free and open access by the Master's Theses and Graduate Research at SJSU ScholarWorks. It has been accepted for inclusion in Master's Theses by an authorized administrator of SJSU ScholarWorks. For more information, please contact scholarworks@sjsu.edu. 


\section{INFORMATION TO USERS}

This manuscript has been reproduced from the microfilm master. UMI films the text directly from the original or copy submitted. Thus, some thesis and dissertation copies are in typewriter face, while others may be from any type of computer printer.

The quality of this reproduction is dependent upon the quality of the copy submitted. Broken or indistinct print, colored or poor quality illustrations and photographs, print bleedthrough, substandard margins, and improper alignment can adversely affect reproduction.

In the unlikely event that the author did not send UMI a complete manuscript and there are missing pages, these will be noted. Also, if unauthorized copyright material had to be removed, a note will indicate the deletion.

Oversize materials (e.g., maps, drawings, charts) are reproduced by sectioning the original, beginning at the upper left-hand corner and continuing from left to right in equal sections with small overlaps. Each original is also photographed in one exposure and is included in reduced form at the back of the book.

Photographs included in the original manuscript have been reproduced xerographically in this copy. Higher quality $6^{\prime \prime} \times 9^{\prime \prime}$ black and white photographic prints are available for any photographs or illustrations appearing in this copy for an additional charge. Contact UMI directly to order.

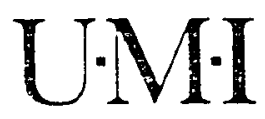

University Microfilms International A Beil \& Howel: Information Company 300 North Zeeb Road. Ann Arbor. MI 48106-1346 USA 

Order Numbar 1345823

A retrospective study to determine the relationship between selected hemocomponents, ultrafiltration, and body temperature

\author{
Smith, Nancy A., M.S.N.
}

San Jose State University, 1991

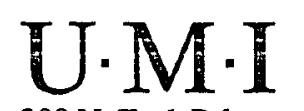

300 N. Zeev Rd.

Ann Arbor, MI 48106 

A RETROSPECTIVE STUDY TO DETERMINE THE

\author{
RELATIONSHIP BETWEEN SELECTED \\ HEMOCOMPONENTS, ULTRAFILTRATION, \\ AND BODY TEMPERATURE
}

\begin{abstract}
A Thesis
Presented to

The Faculty of the Department of Nursing

San Jose State University
\end{abstract}

In Partial Fulfillment

of the Requirements for the Degree

Master of science

By

Nancy A. Smith

August, 1991 
APPROVED FOR THE DEPARTMENT OF NURSING

Persy ithicer

Terry Wf Milier, Ph.D., R.N.

Siendeth C. Sut-

Elizabeth 0. Dietz, EdJD., R.N., C.

$\frac{i \text { whitur. }}{\text { Christine A. Hooper, M.S., R.N. }}$

APPROVED EOR THE UNIVERSITY

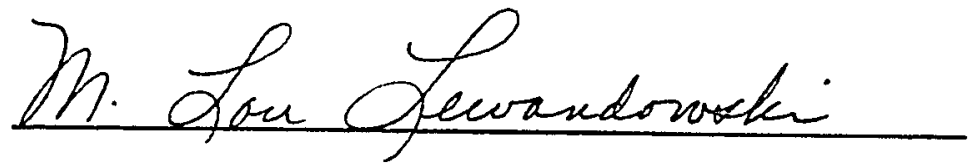




\section{ABSTRACT \\ A RETROSPECTIVE STUDY TO DETERMINE THE \\ RELATIONSHIP BETWEEN SELECTED \\ HEMOCOMPONENTS, ULTRAFILTRATION, \\ AND BODY TEMPERATURE \\ by Nancy A. Smith}

Hemodialysis is the removal of waste material from the blood through filtration of the blood using a semipermeable membrane. This complex process requires a hemodialysis machine and a dialyzer to remove the toxins. Reuse of the dialyzer was started for economic reasons. Currently accepted benefits of dialysis reuse include: (a) the decreased incidence of first use syndrome, (b) a decrease in the abnormal stimulation of the body's immune system, and (c) higher levels of oxygen. Disadvantages include the possible development of an antibody to red blood cells if formaldehyde disinfection is incomplete, accidental dialysis on someone else's dialyzer, and an increased possibility of developing a pyrogen reaction.

This paper reflects the data from one hemodialysis facility in central California. Records were reviewed for a six-month period. Four measures were noted: serum urea nitrogen, serum creatinine, ultrafiltration coefficient, and the change in the patient's body temperature before and after the treatment. The study was retrospective. 
ACKNOWLEDGEMENTS

Thank you to my husband, Glenn, and

children, Kerri, Zachariah, Joshua, and

Sara, for their support and

encouragement. 
TABLE OF CONTENTS

Chapter

Page

1. INTRODUCTION . . . . . . . . . . . . . 1

The Problem and Research Questions . . . . 4

Purpose and Need . . . . . . . . . . 5

Definition of Terms . . . . . . . . . 7

Setting and Sample . . . . . . . . . 9

Research Design . . . . . . . . . . 11

Scope and Limitations . . . . . . . . 13

2. CONCEPTUAL FRAMEWORK AND REVIEW OF RELATED

LITERATURE . • . . . . . . . . . 15

Scope of Nephrology Nursing Practice . . . 15

Standards of Nephrology Nursing Care . . . 16

Background Information . . . . . . . . 18

Review of Related Literature . . . . . . 20

3. THE METHOD . . . . . . . . . . . . 28

Design . . . . . . . . . . . . . 28

Sample . . . . . . . . . . . . . 29

Procedure . . . . . . . . . . . 30

Data Collection . . . . . . . . . . 32

4. ANALYSIS AND INTERPRETATION OF DATA . . . . 35

Analysis . . . . . . . . . . . 35

Interpretation . . . . . . . . . . 37

5. CONCLUSIONS AND RECOMMENDATIONS . . . . . . 39

Conclusions . . . . . . . . . . . . 39 


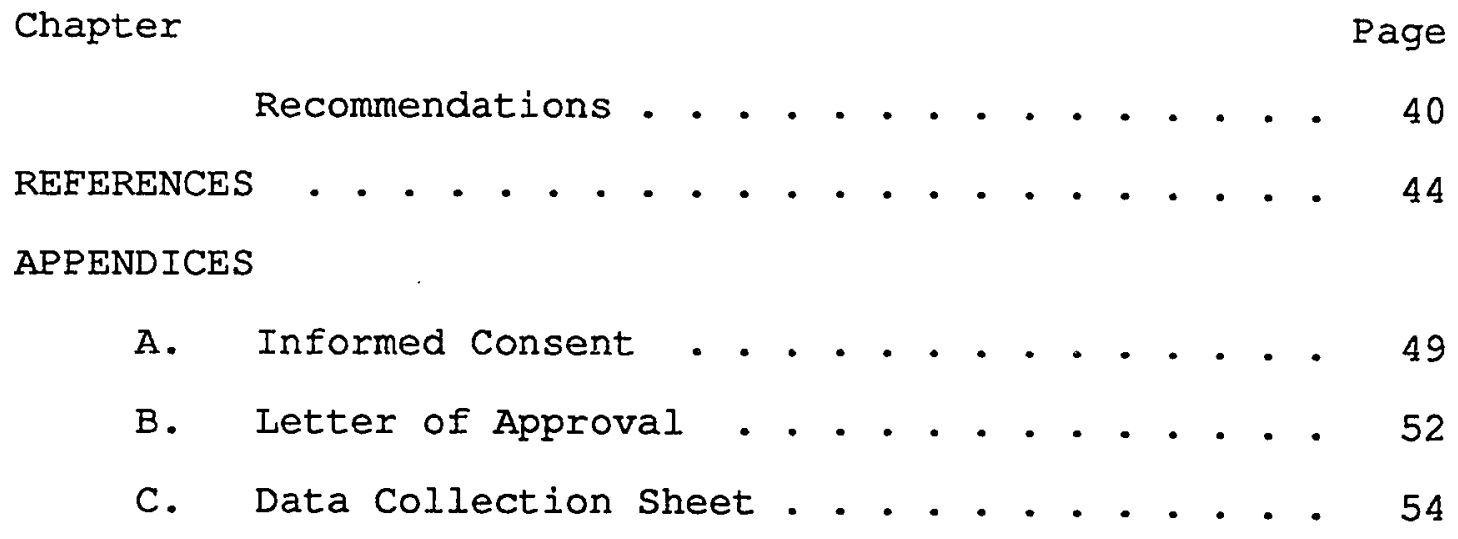




\section{LIST OF TABLES}

Page

Mann-Whitney $\underline{U}$ Data Table . . . . . . . . . . 38 
Chapter 1

INTRODUCTION

Hemodialysis is the removal of waste material from the blood through filtration of the blood using a semipermeable membrane. This complex process requires a hemodialysis machine and a filter to remove the toxins. The first clinically successful dialyzer was developed in 1943. The development of a permanent access to the circulatory system and an effective dialyzer in 1959 signified the beginning of chronic hemodialysis (Papper, 1971).

The dialyzer is an extracorporeal device that removes toxins by diffusion and convective transfer through a semipermeable membrane. New dialyzers contain potentially toxic residue of the manufacturing process, and reused dialyzers contain potentially toxic residue of the reprocessing procedure. Clinicians believe that proper procedures are capable of reducing the levels of these toxins to the point that acute reactions are infrequent. Chronic toxicity has not been documented. Long-term studies must continue to determine the potential toxicity of new and reused dialyzers (National Kidney Foundation, 1984).

Chronic hemodialysis is one treatment modality for people with end stage renal disease. Maintenance dialysis is initiated when the glomerular filtration rate of the kidneys is less than 5 milliliters per minute, and serum 
creatinine levels are greater than 10 milliliters per minute per 100 milliliters. Dialysis also is started in the presence of uremic complications associated with end stage renal disease. These complications include: anemia, bone disease, peripheral neuropathy, uncontrolled hypertension, and congestive heart failure.

Before 1972, there were lower than 200 facilities treating patients with end stage renal disease nationwide. Currently, there are in excess of 1,800 dialysis facilities nationwide. Medicare estimates there are 8,000 new patients reaching end stage renal disease each year. Recently released statistics indicate that there are approximately 105, 958 patients receiving treatment for end stage renal disease; 86,250 are on hemodialysis ("HCFA Release," 1990). Medicare is the primary funding source for patients with end stage renal disease. As the number of patients being treated for end stage renal disease has increased, Medicare has decreased its reimbursement. This has led facilities to look for cost saving measures. The process of dialyzer reuse was developed to meet this need. According to the report of the national workshop on reuse of consumables in hemodialysis, reuse of hemodialyzers saves $\$ 5$ to $\$ 10$ per treatment (Report of National, 1984). The practice of reusing hemodialyzers involves $68 \%$ of all chronic hemodialysis facilities (Biddle, 1987). 
Many physicians feel that reuse has therapeutic advantages over first-use dialyzers because they are less likely to leach dangerous substances into the blood. Reuse lowers the incidence of flushing, shortness of breath, chest pain, fever, sweating, nausea, vomiting, and blood pressure problems associated with the first use of a dialyzer (first use syndrome). Patients reusing their dialyzer have higher blood oxygen levels, and there is less abnormal stimulation of the body's immune system due to blood contact with the dialyzer membrane. Widely disputed among the nephrology community are the therapeutic advantages of dialyzer reuse.

This study examined whether dialyzer reuse affects the performance of the dialyzer. Evaluation of dialyzer performance can be accomplished by measuring several parameters. These include measuring the patient's serum urea nitrogen, serum creatinine, vitamin B-12, insulin clearances, body temperature, and the ultrafiltration coefficient. Dialyzer clearance of uremic toxins and ultrafiltration can be related mathematically to area lost in hollow fiber dialyzers during reuse (Gotch, 1987). Medical records were reviewed for the number of documented pyrogens. Dialyzer reuse can cause a pyrogen reaction if the procedure is not completed according to protocol. 
The Problem and Research Questions

Numerous papers have been published in the past 10 years discussing the advantages and disadvantages of reusing dialyzers. One of the advantages of dialyzer reuse is the lowering of the incidence of first use syndrome. There also is a decrease in the abnormal stimulation of the body's immune system due to blood contact with the dialyzer membrane. Blood levels of oxygen are higher. There is less exposure of the body to small particulate matter left from the manufacturing process or chemicals that leach out of the plastic used to manufacture the dialyzer. There is less exposure to the chemicals used to sterilize the dialyzer after its manufacture (Kant, Pollack \& Goetz, 1987).

The disadvantages of dialyzer reuse include the possible development of an antibody to red blood cells if formaldehyde rinsing is incomplete. Other disadvantages of dialyzer reuse are accidental dialysis on another person's dialyzer, an increased possibility of developing a pyrogen reaction, or infection due to improper reuse procedures. The long term effects of exposure to residual amounts of formaldehyde are unknown (Kant, et al., 1981). Many voluntary regulations have been proposed by the Association for the Advancement of Medical Instrumentation, but few dialysis facilities observe these voluntary standards. This study addressed four research questions: 
1. What is the relationship between the reuse of the dialyzer and the patient's serum urea nitrogen?

2. What is the relationship between the reuse of the dialyzer and the patient's serum creatinine?

3. What is the relationship between the reuse of the dialyzer and the patient's body temperature?

4. What is the relationship between the reuse of the dialyzer and the ultrafiltration coefficient of the dialyzer?

Purpose and Need

Since 1987, dialyzer reuse has been considered the "Standard of Practice" with $68 \%$ of the dialysis facilities reusing (Vlchek, 1987). Successful reuse depends on the consistency of the procedure (Hopkins, 1982). Residual blood in the dialyzer may cause unacceptable decrements in clearance (Hopkins, 1982). Technique varies from facility to facility and even within the facility, depending on the skill of the reuse technician.

The purpose of conducting this study was to examine how dialyzer reuse affects the renal patient's well being. If reuse leads to an increase in the laboratory values, the patient will experience increased uremic symptoms, hypertension, nausea, vomiting, fatigue, loss of appetite, loss of sexual and cognitive function, pruritus, restlessness and seizures. A decrease in ultrafiltration 
can lead to peripheral edema or congestive heart failure (Lancaster, 1984, p. 2). Though renal patients sign a consent form for dialyzer reuse, they are being submitted to dialyzer reuse without fully understanding the side effects of the practice. The information gained from this study can lead to potential applications in the clinical setting which in turn may benefit the renal patient's well being.

The need to study this issue is supported by the fears expressed by patients about reuse. They have unanswered questions about the procedure and its effect on their quality of life (Vlchek, 1987). As reuse is being practiced without sufficient information, this investigator has observed fear leading to resentment and anger usually directed at the nursing staff. Hildegard Peplau describes six different nursing roles emerging from the nurse-patient relationship. Two of these are important when dealing with the practice of dialyzer reuse. As a resource person, the nurse provides specific information and answers questions relevant to the patient treatment plan. As a teacher, the nurse must build on what the patient knows and what he needs to know to make informed decisions about his care (Marriner, 1986, p. 183-184). Nurses need to conduct studies that will help to answer the renal patient's questions about the effects of dialyzer reuse. 
Definition of Terms

For the purpose of this study, the following definitions were used. These definitions are operational and were derived from the Review of Hemodialysis (Gutch \& Stoner, 1983), a standard teaching textbook for hemodialysis personnel:

1. Clearance is a mathematic expression of the rate at which a given substance is removed from a solution. It is the number of milliliters of solution completely cleared of a given solute at one minute.

2. Dialysate is the solution used in the dialysis process.

3. Dialyzer is an extracorporeal device that changes the chemical composition of the body by diffusion and convective transfer of substances through a semipermeable membrane which functions within clinically acceptable rates of water and solute transport.

4. Dialyzer Reuse is the practice of reusing dialyzers that have been cleaned and stored with a disinfectant for use again on the same patient for purposes of hemodialysis.

5. Diffusion is the movement of molecules from an area of greater concentration to one of lesser concentration.

6. End Stage Renal Disease is an irreversible 
condition in which the individual no longer has sufficient renal function to maintain an acceptable clinical state without renal replacement therapy. The determination of end stage renal disease is usually made through a combination of laboratory data, clinical signs, and/or direct visualization of renal tissue obtained by biopsy.

7. First Use Syndrome is associated with the first use of a dialyzer and can include nausea, vomiting, wheezing, chest pain, fever, flushing, sweating, hypotension, and death.

8. Laboratory Values are the measurement of patient's blood with standardized normals. For this paper, serum urea nitrogen and creatinine are monitored every other week.

9. Nephrology nursing is a specialized area of nursing focused on the health needs of individuals and their families who are experiencing the real or threatened impact oI renal disease.

10. Patient is a person who has reached end stage renal disease and elected hemodialysis as the treatment modality.

11. Pyrogen is a fever causing agent of bacterial origin.

12. Reverse osmosis (RO) is the process of removing almost all solutes from a solution by applying high pressure on it against a membrane permeable only to the solvent; used 
to purify water.

13. Serum Creatinine is Iiberated from muscle tissue and excreted by the kidneys at a constant rate. A normal serum creatinine is 1 to 2 milligrams per 100 milliliters.

14. Serum Urea Nitrogen (BUN) is a chemical determination of the amount of nitrogen derived from urea, present in the blood. Actual urea is 2.2 times the BUN value. Normal BUN is 9 to 15 milligrams per deciliter.

15. Semipermeable membrane is a sheet or tube of material that has submicroscopic pores, that allow particles to pass through it in either direction.

16. Transmembrane Hydrostatic Pressure (TMP) is the net sum of the average pressure of the blood entering and leaving the dialyzer minus the average pressure of the dialysate entering and leaving the dialyzer.

17. Ultrafiltration Coefficient (KUE) for a dialyzer is the amount of water renoved from the blood during a given period at a specified pressure. It is expressed in milliliters per hour of mercury of transmembrane pressure.

18. Ultrafiltration is filtration by a pressure gradient between two sides of a porous material. Setting and Sample

The setting for this study was a 13-station outpatient dialysis facility, Iicensed by the California Department of Licensing and Certification to provide hemodialysis services 
to patients with end stage renal disease. To retain its license, the facility must comply with Title 22, California Code of Regulations. Each facility is inspected yearly by the California Department of Licensing and Certification. Staffing ratios, procedures for training new personnel, performing the dialysis procedure, long and short term patient care plans, and the maintenance of medical records are examples of the topics covered in Title 22 .

This dialysis facility is staffed with six registered nurses and five patient care technicians. Title 22 mandates one staff member for three patients. This dialysis facility currently provides hemodialysis to 39 patients each day.

The sample was a non-randomized convenience sample of 25 adult patients. They were divided into two groups, those that reused their dialyzer, and those that did not reuse. The reuse group included 21 patients; 4 patients did not reuse because they were positive for the Hepatitis $B$ Antibody and dialyzed in the isolation room. Infectious disease protocol eliminates them from reuse. The patients were selected because they have their serum urea nitrogen and serum creatinine drawn every six treatments. The patients dialyze three times each week for 3 to 4 hours each treatment. They are all of the patients of one nephrologist.

This dialysis facility has practiced reuse since it was 
opened 4 years ago. Their reuse protocol follows California Standards, which recommend that each dialyzer be discarded if: (a) the fiber bundle volume is less than $80 \%$, or (b) the dialyzer's external casing is not intact. Dialyzer reuse is not employed in patients who are suspected of having an active systemic infection, or are positive for Hepatitis B Antigen. In this facility, reuse is done manually. Each dialyzer is filled with water before use, and the initial volume recorded. After cleaning, the volume is again measured. If the volume is less than $80 \%$, the dialyzer is discarded.

\section{Research Design}

This study was exploratory in nature and used a retrospective descriptive design. This design was chosen because the study determined whether dialyzer reuse, the independent variable, affected the dependent variables of serum urea nitrogen, serum creatinine, the ultrafiltration coefficient, and body temperature.

The advantages of a retrospective study include: (a) increased flexibility when investigating complex relationships among variables, (b) the presence of a control group, (c) the potential practical application in the clinical setting, and (d) the foundation for further studies (LoBiondo-Wood \& Haber, 1986, p. 132-133). The major disadvantage to this type of study is the possibility that 
an extraneous variable was the cause of the differences between the two groups and not the assigned independent variable.

The consent form was designed by the investigator who sought permission from the potential subjects for participation in the study (Appendix A). The form was designed to insure that the patients understood that they were granting a review of their medical records and not participating in experimental therapy. Therefore, permission was obtained from (a) the patient, (b) the medical director of the facility, and (c) Human Subjects Committee of San Jose State University.

After written consent was received, patients' records were reviewed to determine if increases in serum urea nitrogen and serum creatinine correlated to the number of times a dialyzer was reused. The ultrafiltration coefficient was calculated for each treatment and compared to the manufacturer's stated ultrafiltration coefficient. The data was collected both for the patients that reused and those that did not reuse their dialyzer. The Mann-Whitney $U$ was used to analyze the data. The data for each patient were compared between the two groups. The number of documented pyrogens also was noted.

The data for this study were collected from treatment records for the 6 month period July 1, 1988 through 
December 31, 1988. This time frame was chosen because there were no new personnel being trained to perform the reuse procedure. There were no changes in equipment or disinfect:ants.

Scope and Iimitations

The limitations of this study are as follows:

1. The type of dialyzer used in the study may prevent the results pertaining to its reuse from being generalized to other types of dialyzers.

2. The protocol for cleaning the dialyzer in the study's facility also may limit generalizability to this facility only.

3. The use of a convenience sample of only 25 patients, with four of these patients making up the control group, also may limit the generalizability.

4. The sample was not random within the facility nor random within the dialysis community as a whole.

5. Dialyzer reuse has been the policy at this facility since it opened. No comparative studies can be made to determine if there is any change in the patient's well being with and without reuse.

6. Laboratory values can be affected by diet, exercise, and the general health status. It is beyond the scope of this study to control for these potentially intervening variables. These data are representative of the 
effects of dialyzer reuse on the laboratory values, ultrafiltration coefficient, and body temperature of the patients dialyzing at this facility only.

7. The use of patients currently dialyzing in the facility without reviewing the records of deceased patients and the cause of death may limit the generalizability. 
CONCEPTUAL FRAMEWORK AND REVIEW OF RELATED LITERATURE

Scope of Nephrology Nursing Practice

Nephrology nursing is concerned with adult and

pediatric patients and their families experiencing the real

or threatened impact of acute or chronic renal failure. Nephrology nurses are responsible for the prevention of disease and the care and assessment of the health needs of these families. The nurse must realize that these patients are not extensions of the dialysis machine, but must be viewed holistically (Careers in Nephrology Nursing, 1989). These people have multiple medical, nursing, and psychosocial problems which include: hypertension; cardiac disease; bone disease; growth retardation in children; fluid, electrolyte, and metabolic imbalances; diabetes; nutritional deficiency or excesses; episodes of depression; alterations in body image; and sexual dysfunction. Many patients and their families learn to cope with these problems with the help of skilled nurses.

Physician presence in the hemodialysis unit is minimal. The nurse must be competent in assessment skills, basic nursing procedures, priority setting, nursing diagnosis, and decision-making. Even in the acute setting, after the physician has evaluated the patient, the nurse has the responsibility for providing the treatment. 
The scope of practice for nephrology nursing is established by the American Nephrology Nurses Association. Nephrology nurses must focus on the provision of replacement therapy, and teaching self care. They assist patients in making informed choices regarding the type and location of therapy and the prevention of intercurrent illnesses or complications associated with the various modalities of therapy.

There is a need for research to be done in all areas of renal replacement therapy. Nephrology nursing research offers improvement of and advances in clinical practice for the benefit of the patients and their families. The safety and effectiveness of the practice must be established through an adequate research base and systematic investigation (Baldasseroni, 1984). Standards of Nephrology Nursing Care

Dialyzer reuse involves cleaning the dialyzer and storing it in a disinfectant until the patient's next treatment. The dialyzers are labeled by the manufacturer "For Single Use Only." Nephrology nurses must not only understand the principles of reuse, but must provide comprehensive education for all patient care personnel on the effects inadequate sterilization would have on the patients. It is the responsibility of nursing staff initiating dialysis to verify that the level of disinfectant 
is low enough to meet recommended standards. This must be visually verified by the patient by testing the normal saline in the dialyzer for the presence of disinfectant. The negative result is documented on the treatment record by both the nurse and the patient. The nurse must provide adequate staff education with respect to the patient consequences of formaldehyde infusion. Nurses also are responsible for staff education concerning the harmful effects of formaldehyde exposure, both by skin contact and in the atmosphere. Emphasis must be placed on the strict adherence to safety precautions. Nurses also may be responsible for performing and documenting formaldehyde air-testing, and enforcing the use of all available safety equipment when performing reuse procedures. It is the responsibility of the nursing staff to see that the volume testing of the reprocessed dialyzer has been done consistently and accurately (Baldasseroni, 1984).

The nurse also must educate the patients on the advantages and disadvantages of reuse (Careers in Nephrology Nursing, 1989). The patient must understand the procedure, and what safety and quality control measures are being employed. Regardless of who presents the consent form to the patient, it is the responsibility of the nursing staff to discuss reuse completely with the patients since they are the ones to whom the patients entrust their care on a daily 
basis. Dialyzer reuse is an issue that needs further research because many patients, physicians, and nurses question whether reuse is safe and effective.

Background Information

Renal failure is defined as irreversible kidney disease, causing abnormalities in the internal environment. Renal failure necessitates treatment with dialysis or transplantation for survival (Lancaster, 1984). The symptoms and physiochemical changes that occur are referred to as uremia. These changes are related to fluid and electrolyte abnormalities, disordered regulatory function, and the accumulation of uremic toxins which lead to physiologic changes and alters the functions of various organ systems (Papper, 1971, p. 5). The physical principles involved in hemodialysis fall into two broad categories: (a) solute removal (mass transfer), and (b) fluid removal. Solutes are removed from the body by diffusion across a semipermeable membrane that separates the blood compartment from the dialysate compartment of the hemodialyzer. When equilibrium is reached, diffusion ceases.

Blood and dialysate are pumped in opposite directions on opposite sides of the semipermeable membrane in a closed compartment. The blood contains excess metabolic waste and electrolytes. The dialysate contains the ideal plasma concentration of electrolytes. Mass transfer is observed 
because of the difference in concentration of electrolytes. Metabolic waste moves into the dialysate, and electrolytes move in both directions to maintain equilibrium. Red blood cells, white blood cells, proteins, bacteria, and viruses are too large to pass through the pores of a semipermeable membrane.

Urea nitrogen is an end product of protein metabolism and is a major component of urine. Blood urea nitrogen is a measurement of the level of urea in the serum. Urea nitrogen is normally excreted by the kidneys to maintain a serum level of 20 to 25 milligrams per 100 milliliters. The serum urea nitrogen provides a determination of renal function, or adequacy of dialysis. There are other variables that can elevate the serum urea nitrogen, limiting its usefulness. These include high protein diets, blood in the gastrointestinal tract, or a catabolic state (Lancaster, 1984, P. 108). Creatinine is Iiberated from muscle tissue at a constant rate and is excreted by the kidneys at the same rate. No other variables affect serum creatinine. Patients on hemodialysis have their laboratory values monitored at least monthly, on the first wednesday or Thursday of the month. A serum urea nitrogen greater than 90 or a serum urea nitrogen:creatinine ratio greater than 10:1 indicates a need for increased dialysis (Richards, 1986, p. 242). Body temperature pre-dialysis may be 
decreased if the serum urea nitrogen is elevated to any degree. Post dialysis, the temperature is usually higher because the serum urea nitrogen has decreased. A temperature greater than $99.6^{\circ} \mathrm{F}$ indicates sepsis. Blood and dialysate cultures should be obtained following the facilitie's protocol. Positive blood and dialysate cultures indicate a pyrogen reaction and the need to disinfect the water system and reuse equipment.

Each patient has an ideal body weight established by the nephrologist. Because these patients have little residual kidney function, they gain weight between dialysis treatments. This weight is caused by fluid retention. Using a mathematical formula, the transmembrane pressure necessary to reach the target weight can be calculated (Richards, 1986, p. 246).

Review of Related Literature

Dialyzer reuse was begun during the eariy period of hemodialysis because of the time required to build the hemodialyzers. The first dialyzers were built at the dialysis facility by the staff. Early workers discovered that the dialyzers could be rinsed and filled with disinfectant and reused two or more times. The dialyzer is an extracorporeal device that changes the chemical composition of the body by diffusion and convective transfer of substances between the blood and a solution of chemicals 
through a semipermeable membrane. This membrane must function within clinically acceptable rates of water and solute transfer (Simesen, 1985).

Since 1986, the Centers for Disease Control (CDC), in collaboration with the Health Care Financing Administration (HCFA), has surveyed 1,350 chronic hemodialysis facilities in the United States to ascertain practices associated with reuse and the frequency of pyrogenic reactions and septicemia among patients. The results of this survey indicated that problems associated with disease transmission and hemodialyzer reuse have been almost exclusively due to inadequate processing (Alter, Favero, Coleman \& Bland, 1988). During a pyrogen reaction, large numbers of organisms are infused into the patient via the venous return from the dialyzer. In one Centers for Disease Control report, 16 of 32 patients grew pseudomonas cepacia from their blood following an inadvertent omission of a "step in the sterilization and storage procedure," a quality control omission. Thirteen of the 16 became clinically ill. The symptoms of chills, fever, hypotension, nausea, and myalgia began within the first hour of dialysis and lasted several hours (Ogden, 1986).

The Centers for Disease control has documented by culture non-tuberculous mycobacteria in $70 \%$ of city water samples and $48 \%$ of dialysis facility water, which may 
multiply in water treatment and distribution equipment. They are highly resistant to the effects of most disinfectants, including formaldehyde. These characteristics of the mycobacteria species, combined with inadequate quality control of the formaldehyde used in the reprocessing of dialyzers, resulted in 27 cases of non-tuberculous mycobacteria infection among 142 patients at two Baton Rouge outpatient centers. Clinical illness was associated with these infections. Symptoms of elevated temperature, rigors, nausea, vomiting, and diarrhea were reported. To what extent these infections contributed to patient mortality is unknown. Thirteen of 27 died within less than a year after their infections (Ogden, 1986). The Centers for Disease Control findings generally implicate infrequent sterilization of the reverse osmosis unit used to purify raw city water. Other causes of pyrogenic reactions are a disinfectant that is too dilute used in the reprocessing and storage of the dialyzers, or the use of raw water to prepare the disinfectant. Frequent monitoring of bacteria levels and the maintenance of these levels below 200 colonies are essential (Ogden, 1986). Recommendations are for a $4 \%$ formaldehyde solution rather than the $1.8 \%$ solution currently used by most facilities (Senator, Clinicians, 1987). In addition, pre- and post dialysis body temperatures are taken on all patients. 
Standard protocols for suspected pyrogen reactions are blood and dialysate cultures. If the same bacteria are found in the blood and dialysate cultures, a pyrogen reaction has occurred.

Vanholder, Piron, Cutber, and Vermaereke (1987), studied serum urea nitrogen, serum creatinine, B-12, and insulin clearances, as well as ultrafiltration capacity, during first, third, and sixth reuses for a 2 year period. The dialyzers were reprocessed using an automatic system that allows for dialyzer quality control. This equipment tests for ultrafiltration capability and pressure leaks. They determined that there were no significant changes in laboratory values or ultrafiltration with six reuses using a cuprophane dialyzer. This was not true with the polyacrylinetricle dialyzer; reuse was limited to three due to increasing laboratory values. Another study done at the University of Hawaii (Simesen, 1985) concluded that there were no significant changes in the clearance rate at 1,5 , and 10 reuses. There was a drop in ultrafiltration rate of $15 \%$, leading to the establishment of a maximum of 10 reuses. A study done by Dr. Frank Gotch at Davies Medical Center (Gotch, 1987) reported that if the measured loss in area of the dialyzer is less than 20\%, the mean loss in clearances of all the solutes of interest, from phosphate to urea, will be linear and will be $20 \%$ for an area loss of 
20\%. However, with the solutes of established clinical importance in the dialytic management of uremia, reusing the dialyzer until the area loss reaches $20 \%$ will have a minimal effect on clearance (Gotch, 1987). Numerous other studies have achieved similar results (Craske, Dabrowiecki \& Kennedy, 1982; Gotch, 1985; Hoenich, Johnston \& Buckley, 1984).

Based on the studies done by Gotch (1985), Craske, et al. (1982), Hoenich, et al. (1984), the Association for the Advancement of Medical Instruments has established guidelines for the reuse of hemodialyzers. The Advancement of Medical Instrumentation is recognized by the Health Care Financing Association and the Centers for Disease Control as the leading researcher and authority on reuse of dialyzers. The committee was comprised of physicians, nurses, patients, and representatives of the manufacturers of dialyzers and reuse equipment. They have designed voluntary guidelines. California has passed legislation that makes them mandatory.

The Association for the Advancement of Medical Instrumentation recommends that urea clearance should be used as the criteria for rejecting a dialyzer since the clearance of low molecular weight molecules decreases clinical symptoms of uremia (Recommended Practice for Reuse of Hemodialyzers, 1986). They have suggested a \pm 108 change in serum urea nitrogen as acceptable. They also recommend 
that the in vivo ultrafiltration coefficient be $\pm 20 \%$ of the manufacturer's ultrafiltration coefficient.

Manufacturers of new dialyzers use different techniques to produce dialyzers, but must meet certain standards of safety. These standards are validated by studies of small samples. Standards of performance are established for each dialyzer based on manufacturing specifications and supported by validation studies. Manufacturers do not do any studies on the reuse of a dialyzer. Supporters of reuse feel that the gases used to sterilize the dialyzer or the organic chemicals used to manufacture the plastic fibers cause allergic-type reactions (Simesen, 1985). There are many reports of first use syndrome where, during the first minutes of a treatment using a new dialyzer, patients will experience tightness in the chest, wheezing, itching, and possible death.

Supporters of reuse, such as Gotch (1987) and Chenoweth, Cheung, Ward, and Henderson (1983), point to the consistently documented leukopenia of patients using new cuprophane hollow fiber dialyzer. There is also an increase in the plasma level of the bioactive peptides $\mathrm{C} 3 \mathrm{a}$ and $\mathrm{C5a}$ during initial phases of the treatment (Chenoweth, et al., 1983). Hemodialysis with dialyzers exposed to formalin disinfection prior to blood contact produced 20 to $30 \%$ less peripheral blood leukocytes. Reused dialyzers also differed 
from either new or formalin fixed dialyzers, in that c3b antigen could be detected within them even after extensive washings. These observations suggest that $\mathrm{C} 3 \mathrm{~b}$ deposition on the cellulosic membrane surface during first use decreases the complement activation potential of cuprophane dialyzers when they are subsequently reused (Chenoweth, et al., 1983). The development of hypoxemia and pulmonary dysfunction during dialysis has been attributed to intrapulmonary leukocyte entrapment caused by the activation of the complement system (Vanholder, et al., 1987). The studies done by Chenoweth, et al. (1988) and Vanholder, et al. (1987) have shown that there are significant differences in intradialytic alveolar oxygen tension (pao2) during first use and reuse. During first use, there was a decline in lung diffusion capacity and transfer factor. This phenomenon was prevented with reuse. The data from their studies show a direct relationship between intradialytic complement activation and leukocyte pulmonary sequestration and pulmonary dysfunction (Chenoweth, et al., 1983; Vanholder, et al., 1987). Complement activation is one aspect of blood-materials interactions and may be considered an index of biocompatibility. Products of complement activation have several biological actions, leukoaggregation, histamine release from most cells, and an increase in capillary pulmonary permeability which may 
contribute to adverse clinical and biological changes (Hakim, Fearson \& Lazarus, 1984). Hakim states that indirect evidence reported by his colleagues indicates that chronic dialysis with complement activating surfaces may be associated with increased mortality and morbidity in dialysis patients (Hakim, et al., 1984).

The most commonly expressed concern of patients regarding reuse relates to the acute or long-term effects of formaldehyde, the most commonly used disinfectant. Acute toxicity includes discomfort at the venous return and the formation of anti-N-like antibodies resulting from formaldehyde residuals greater than 10 and up to 100 parts per million (ppm). Chronic toxicity from small intravenous infusions of formaldehyde had not been reported in animals or man (Ogden, 1986).

A recently published crossover study concluded that there were no differences between patients treated with new and reused dialyzers. The variables studied included body temperature, blood leaks, serum creatinine, and the clinical symptoms of pruritus, cramps, nausea, headache, chest pain, or fatigue (Churchill, et al., 1988). It is necessary for each dialysis facility to study the performance of their reprocessed dialyzer to determine whether it meets functional criteria. For this reason, this retrospective study was conducted. 


\section{Chapter 3}

THE METHOD

Design

This retrospective study compared two groups of patients receiving renal dialysis. One group of patients reused their dialyzers, while the other group did not. The groups were compared in terms of their serum urea nitrogen levels, serum creatinine levels, ultrafiltration coefficients, and pre- to post-dialysis changes in body temperature. Twenty-five patient's records were reviewed for a 6 month period from 1 July 1988 to 31 December 1988. The laboratory values were obtained every sixth treatment by drawing 6 milliliters of blood prior to the initiation of dialysis, and placing it in a serum separator chemistry tube. After the blood clotted, it was centrifuged for 5 minutes and transported to the laboratory. The serum was placed on slides and put into a Kodak analyzer. The results were received electronically within minutes. The ultrafiltration coefficient was calculated for each treatment and compared to the manufacturer's stated ultrafiltration coefficient. The patient's body temperature was recorded pre-dialysis and the degree of change postdialysis was noted. The records were reviewed to note how febrile reactions were documented. There were no equipment changes or new personnel during the review period. 
The subjects' rights were protected by informed consent, which each patient signed (Appendix A). Approval to review the records was received from the Medical Director of the facility (Appendix B). Approval for the study was granted by the Human Subjects Committee of San Jose State University.

\section{Sample}

The patients in this study had end stage renal disease and had chosen hemodialysis as their mode of therapy. They were being treated at a free standing clinic located on the Central California coast. Currently, this dialysis clinic treats 85 patients and has four nephrologists. There are three other dialysis clinics on the Central Coast, each has 40 to 60 patients and two nephrologists.

The sample was a non-randomized convenience sample of 25 adult patients. They were all of the patients of one nephrologist. They had their laboratory values drawn every sixth treatment. They were divided into two groups, 21 that reuse, 4 that do not reuse their dialyzers. Each patient was assigned a number to maintain confidentiality. Their sex and age were recorded to better describe the sample and possibly guide further research. The age range was 20 to 70 years, the average age was 50 years; 14 patients were male, 11 female. 
Procedure

Prior to the initiation of each dialysis, the patient's vital signs were recorded. Body weight in kilograms was recorded pre and post dialysis using a scale built into the floor. The scale accommodated wheelchairs to allow non-ambulating patients to be weighed. Any food or beverage the patient planned to consume during treatment also was weighed. The scale was calibrated 31 January 1988 by the Department of Weights and Measures to insure accuracy; this is done annually. Body temperature was taken orally, using temp-a-dot strips pre and post dialysis. A radial pulse was taken and the blood pressure recorded using a mercury sphygmomanometer. These measurements were taken on the patient's dominant forearm. The hemodialysis access was usually placed in the non-dominant extremity. No blood pressure or needle sticks in blood vessels of the non-dominant arm were permitted, to prevent unintentional injury to the blood vessels.

The length of time the thermometer remained in the patient's mouth, the length of time the pulse was counted, and the method of auscultating the blood pressure were dependent on the personnel initiating the dialysis treatment. The staff members initiating the dialysis treatment calculated what transmembrane pressure (TMP) was needed to reach the patient's ideal body weight. For 
example, if the physician designated 50 kilograms (kg) as the ideal body weight and the patient's weight pre-dialysis was $52.5,2.5 \mathrm{kilograms}$ of fluid was to be removed. The length of the treatment was divided into the number of cubic centimeters (cc) to be removed, divided by the manufacturer's stated ultrafiltration coefficient:

$$
\frac{2500 \mathrm{cc}}{3 \text { hours }} \text { to be removed }=\frac{833 \mathrm{cc} / \mathrm{hr}}{4.2 \text { ultrafiltration coefficient }}=198 \text { Total TMP }
$$

To establish some control over dialysis facilities that reuse, the State of California Licensing and Certification Department requires reuse records to be kept. The records must include date, serial number, use number, fiber bundle volume initially, fiber bundle volume after each use, percentage of clotted fibers, size of dialyzer, reason for discard, and initials of the technician performing reuse. The dialyzers must be discarded when $20 \%$ of the fibers are clotted.

Prior to the first use, the baseline fiber bundle volume was determined. The dialyzer was then filled with formaldehyde, so even patients that did not reuse were not exposed to the chemicals and plastic residue of the manufacturing process. If the dialyzer was to be reused, when the dialysis treatment was completed, the dialyzer was processed according to the following protocol. The blood compartment was rinsed with purified water (approximately 
2,000 milliliters at 20 pounds per square inch). Following this, reverse ultrafiltration was carried out with purified water (1,000 milliliters).

Fiber bundle volume was then determined and dialyzers with volumes less than 80 \% of baseline were discarded. The dialyzer was then stored in a $4 \%$ formaldehyde solution, which was used to fill both the blood and dialysate compartments. Prior to the next use, the formalin was removed, both by dialysis and washing of the blood path with sterile normal saline (500 milliliters). Finally, the dialyzer effluent was analyzed with Formalert reagent to insure that residual formalin content was one part per million. Each dialyzer was stored for 24 to 36 hours before reuse.

A Cobe Century 2Rx Dialysis Control Unit was used with standard dialysate flow rates of 500 milliliters per minute in the single pass mode. Dialysate temperature was maintained at $34^{\circ}$ to $38^{\circ} \mathrm{C}$. Blood flow rates were 200 to 250 milliliters per minute. Dialyzers made of cuprophane membrane were used exclusively in this dialysis facility.

Data Collection

After the patients' written consent was obtained (Appendix A), their medical and reuse records were examined. A data collection sheet was designed by the researcher (Appendix C). This sheet had spaces for noting patient 
number, age, sex, the dialyzer prescribed by the nephrologist, manufacturer's stated ultrafiltration coefficient, serum urea nitrogen, serum creatinine, temperature pre dialysis, the actual ultrafiltration coefficient, and the use number of the dialyzer. The comment column was used to note the degree of change in the patient's temperature post dialysis, whether pyrogen protocol was followed for temperatures greater than $99.6^{\circ} \mathrm{F}$, or the medical records documented other causes for the increased temperature, such as wound infections, viral or bacterial syndromes, or transfusion reactions.

This collection sheet was designed to organize the data for each patient. Each patient had 65 to 79 treatments during the 6 month period reviewed. Organizing the data needed for this study would not have been feasible without a collection sheet. The daily treatment logs were reviewed to determine what the ultrafiltration coefficient was for each treatment. These data were compared to the manufacturer's stated ultrafiltration coefficient, and listed on the data collection sheet as stated KUF, and actual KUF. There were three different size dialyzers used in this facility, cobe Hollow Fiber 100, 120, and 140. This information was listed on the data collection sheet to ensure that the appropriate ultrafiltration coefficient was used in the calculation of the ultrafiltration coefficient. 


\begin{abstract}
Serum urea nitrogen and serum creatinine levels were recorded. These hemocomponents were drawn every sixth treatment. The temperature pre dialysis and the degree of change at the completion of the dialysis were also recorded. The reuse records were used to record the number of each use in relation to the ultrafiltration coefficient, body temperature, and, when applicable, the serum urea nitrogen and serum creatinine.
\end{abstract}




\section{Chapter 4 \\ ANALYSIS AND INTERPRETATION OF DATA}

Analysis

The Mann-Whitney $\underline{U}$ statistic was computed for each of the dependent variables because of a small sample size, $\underline{N}=25$. Specifically, $\underline{N}_{\mathrm{a}}=4$ for the non-dialyzer reuse group, and $N_{b}=21$ for the dialyzer reuse group. The investigator originally planned to analyze the data with an analysis of variance, a sample of four in one group did not meet the assumptions of a parametric test.

The Mann-Whitney $\underline{U}$ test is a nonparametric alternative for the $t$ test for the difference between two independent means. The test is often applied in situations in which two samples are drawn from the same population, but different "treatments" are used on each set (Shott, 1990). The test is also applicable because the samples are of unequal size. For small samples, one or both with $\underline{N}^{\prime}$ s less than eight, the U statistics should be computed using a special Mann-mitney $\underline{U}$ formula, $\left(\underline{U}_{1}=\underline{N}_{\mathrm{a}} \underline{\underline{b}}_{\mathrm{b}}+\left(\underline{N}_{1}\left[\underline{N}_{1}+1\right]\right) / 2-\underline{R}_{1}\right)$ (Guilford \& Fruchter, 1978).

The null hypothesis tested was that the measures of the group that did not reuse their dialyzer would be equal to the group that did reuse their dialyzer. The alternative hypothesis was that the measures of one group would be different (Guilford \& Fruchter, 1978). Group A, the non 
reuse group, was limited in size because it is the policy of this facility for all patients to reuse their dialyzer unless they are positive for Hepatitis $B$, or refuse to reuse their dialyzer. None of the patients refused to reuse dialyzer in the history of this clinic. The control group patients included in this study were antibody positive for Hepatitis $B$ and did not reuse their idalyzers for infection control reasons. They dialyzed in the isolation room used for patients positive for Hepatitis $B$.

There are repeated measures (11 to 77) of the dependent variables, serum urea nitrogen, serum creatinine, ultrafiltration coefficient, and body temperature for each of the 25 patients. The patients had 65-79 hemodialysis treatments during the same period; the ultrafiltration coefficient and temperature were recorded for each treatment. As a variance reduction technique, the repeated measures were averaged for each patient.

For each dependent variable, a single rank order was assigned for all the values in the two samples. The numerically lowest ranks were assigned to the lowest values. The sum of the ranks for each group was then obtained. A two tailed test was applied with an alpha of .10 and with $\underline{N}_{\mathrm{a}}=4$ and $\underline{N}_{\mathrm{b}}=21$. The statistical significance of the departure of the obtained $\underline{U}$ from zero was determined by reference to tables constructed for the Mann-Whitney $\underline{U}$ test. 
The Mann-Whitney $\underline{U}$ table gives a critical value of $\underline{U}=19$. If the data produce a $\underline{U}$ less than or equal to 19, the null hypothesis will be rejected (Guilford \& Fruchter, 1978).

The sum of the ranks for serum urea nitrogen for the non reuse group was $\underline{R}_{\mathrm{a}}=52$; for the reuse group, $\underline{R}_{\mathrm{b}}=273$. The Mann-Whitney $\underline{U}$ was the smaller of the two values obtained (Shott, 1990). As a check for the $U$ calculations, $\underline{U}_{\mathrm{a}}+\underline{U}_{\mathrm{b}}=\underline{N}_{\mathrm{a}} \underline{\underline{N}}_{\mathrm{b}}$ was used (Guilford \& Fruchter, 1978). The $\underline{R}_{\mathrm{a}}$ for the dependent variable of serum creatinine for the non reuse group was 67.5 , the $\underline{R}_{\mathrm{b}}=257.5$. The $\underline{U}_{\mathrm{a}}=26.5$, the $\underline{U}_{\mathrm{b}}=57.5$, the final Mann-Whitney $\underline{U}$ would be 26.5. The sum of the ranks for the ultrafiltration coefficient was $\underline{R}_{\mathrm{a}}=60, \underline{R}_{\mathrm{b}}=265$ with $\underline{U}_{\mathrm{a}}=35$ and $\underline{U}_{\mathrm{b}}=50$. The final Mann-Whitney $\underline{U}$ for the ultrafiltration coefficient was 34 . The fourth dependent variable, body temperature, had a rank sum of $\underline{R}_{\mathrm{a}}=32, \underline{R}_{\mathrm{b}}=263$ with $\underline{U}_{\mathrm{a}}=32$ and $\underline{U}_{\mathrm{b}}=52$. Table 1 illustrates the Mann-Whitney $\underline{\underline{U}}$ values.

\section{Interpretation}

The critical $\underline{U}$ value was 19 (Guilford \& Fruchter, 1978). In all of the calculations, the $\underline{U}$ value obtained was not less than or equal to 19. Because the $\underline{U}$ values obtained are not in the critical region, the null hypothesis was accepted. With an alpha of .10, the data did not provide sufficient evidence to conclude that there was a discernible difference between the dependent variables of the two groups. 
Table 1

Mann-Whitney U Data Table

\begin{tabular}{|c|c|c|}
\hline Variable & $\begin{array}{l}\text { Sum of } \\
\text { the Ranks }\end{array}$ & Mann-Whitney $\Psi$ \\
\hline \multirow[t]{2}{*}{ Serum urea nitrogen } & $\underline{R}_{\mathrm{a}}=52$ & $\underline{U}_{\mathrm{a}}=42$ \\
\hline & $\underline{R}_{\mathrm{b}}=273$ & $U_{b}=42$ \\
\hline \multirow[t]{2}{*}{ Serum creatinine } & $R_{\mathrm{a}}=67.5$ & $\underline{U}_{\mathrm{a}}=26.5$ \\
\hline & $R_{\mathrm{b}}=257.5$ & $\underline{U}_{\mathrm{b}}=57.5$ \\
\hline Ultrafiltration & $\underline{R}_{\mathrm{a}}=60$ & $U_{\mathrm{a}}=34$ \\
\hline coefficient & $\underline{R}_{\mathrm{b}}=265$ & $\underline{U}_{\mathrm{b}}=50$ \\
\hline \multirow[t]{2}{*}{ Body temperature } & $\underline{R}_{\mathrm{a}}=32$ & $\underline{U}_{\mathrm{a}}=32$ \\
\hline & $\underline{R}_{\mathrm{b}}=263$ & $\underline{\underline{u}}_{\mathrm{b}}=52$ \\
\hline
\end{tabular}


Chapter 5

CONCLUSIONS AND RECOMMENDATIONS

Conclusions

This study addressed four questions. They are as follows:

1. What is the relationship between the reuse of the dialyzer and the patient's serum urea nitrogen?

2. What is the relationship between the reuse of the dialyzer and the patient's serum creatinine?

3. What is the relationship between the reuse of the dialyzer and the patient's body temperature?

4. What is the relationship between dialyzer reuse and the ultrafiltration coefficient of the dialyzer?

The results of this study on the reuse of dialyzers indicate that there was no significant difference in the variables studied. Patients who reused their dialyzer did not have a significantly different serum urea nitrogen, serum creatinine, ultrafiltration coefficient, or body temperature than patients that did not reuse their dialyzer.

There were no documented pyrogens during the study period. The post dialysis body temperature of the patients reusing their dialyzer was not significantly different from those patients that did not reuse their dialyzer. This appears to indicate that the percentage of disinfectant and the procedure for reprocessing the dialyzers is adequate. 
As stated earlier, a Centers for Disease Control survey found pyrogenic reaction to be associated with greater than 20 reuses. None of the patients studied at this facility reused their dialyzer more than 20 times.

Recommendations

The limitations of a retrospective study include incomplete laboratory values, lack of baseline information, individual differences of patients, and the presence of uncontrolled extraneous variables. This study had a small sample size. Only general trends can be noted. The data are not transferable to other types of equipment or reuse products. It would not be feasible to use an experimental design because total control is not possible due to the individuality of the patients, the small number of patients that do not reuse, the potential for harm to the patients should the system fail, and the inability to study the patients before they begin to reuse their dialyzers.

If another retrospective study were done in the future, it would be stronger if the sample size for both groups, dialyzer reuse and non reuse was larger. Other facilities using the same dialyzers with similar populations but using automated reuse machines should be studied.

Another limitation of this study was the fact that some of the dialyzers had ultrafiltration coefficients greater than the manufacturer's stated ultrafiltration coefficient 
after 15 reuses. Computer technology is making it possible for the dialysis machines to calculate the ultrafiltration coefficient for each dialyzer every hour. This advance ensures that the patients lose the calculated amount of fluid even with reused dialyzer.

A prospective study should be done in the future. This type of study would be stronger than the retrospective study because of the degree of control that can be imposed on extraneous variables that might confound the data. The advantage of this type of study for the clinical setting is the length of time most patients receive chronic hemodialysis. The staff could be educated about the study to ensure that all necessary data were accurately obtained and recorded for each treatment.

More studies need to be done to document when effectiveness decreases. To date, no universal number of reuses has been established. Some facilities document 50 reuses (Ogden, 1986). It appears that discarding the dialyzer when $20 \%$ of the filters are clotted, or when the usage reaches $20 \%$, would be appropriate until further studies can be carried out to determine the effectiveness of the dialyzer after 20 uses. The rights and obligations of government, practitioners, and patients have caused an emotional debate for years within the nephrology community. The nephrology community has not reached a clear conclusion. 
Similar studies should be done by all facilities practicing reuse. The person performing the reuse procedure has significant impact on the quality of the cleaning and, therefore, on the efficiency of the dialyzer. The facilities need to monitor the patient's laboratory values for increases that correlate with the increasing number of uses. Weights need to be monitored to ensure that the ultrafiltration coefficient is not decreasing as the numbers of clotted fibers increase. Temperature should be monitored and appropriate action taken if a suspected pyrogen occurs. The data should be analyzed for increased numbers of pyrogens or clusters of pyrogens. These studies would allow corrective actions before signifitant detrimental effects were felt by the patients.

The growing practice of reuse makes it essential that the standards of safety and performance be maintained. The patient has the right to expect professional, safe, and effective care at all times. Manufacturers of dialyzers are reluctant to explore the effectiveness of reuse though the director of the Food and Drug Administration has requested such a study (Caplain, 1984). There should be a change in their labeling practices (for one time use only). To date, there has been no positive response. The data provided by physicians with a monetary interest in the provision of dialytic therapy can and has been challenged (Caplan, 1984). 
It is the responsibility of the nephrology community to respond to this challenge. 
REFERENCES 
References

Alter, M. J., Favero, M. S., Coleman, P. I., \& Bland, I. A. (1988). Reuse of hemodialyzers, results of nationwide surveillance for adverse effects of hemodialyzers. Journal of American Medical Association, 260, 2073-2076.

Baldasseroni, A. (1984). Nursing aspects of dialyzer reuse. Journal of Nephrology Nursing, 1(1), 17-19. Biddle, G. (1987). Blood money: TV newscasts irresponsible. Nephrology News and Issues, 1(1), 30. Caplan, A. L. (1984). Dialyzer: To reuse or not to reuse, ethics is the question. American Journal of Nephrology, 4. $128-131$.

Careers in nephrology nursing. (1989). Pitman, NJ: American Nephrology Nurses Board of Directors. Chenoweth, D. E., Cheung, A. K., Ward, D. M., \& Henderson, L. W. (1983). Anaphylatoxin formation during hemodialysis: Comparison of new and reused dialyzers. Kidney International $23(5), 770-774$.

Churchill, D. W., Taylor, D. W., Shimizu, A. G., Beecroft, M. L., Singer, J., Barnes, C. C., Ludwin, D., Wright, N., Sackett, D. I., \& Smith, E. K. (1988). Dialyzer reuse - a multiple cross over study with random allocation to order of treatment. Nephron, 50, 325-331. 
Craske, H., Dabrowiecki, M., \& Kennedy, I. (1982).

Evaluation of dialyzer reuse at Toronto Western Hospital. International Journal of Artificial Organs, $22(6)$, 208-218.

Gotch, F. A. (1985). Mass transport in reused dialyzers. Dialysis and Transplantation, $15(12), 22-26$. Gotch, F. A. (1987). Quality control and dialyzer processing. Dialysis and Transplantation, 16(1), 22-25. Guilford, J. P., Fruchter, B. (1978). Fundamental statistics in psychology and education. San Francisco, CA: McGraw-Hill.

Gutch, C. F., \& Stoner, M. (1983). Review of hemodialysis for nurses and dialysis personnel. St. Louis, MO: C. V. Mosby -

Hakim, R. M., Fearson, D. T., \& Lazarus, J. M. (1984). Biocompatibility of dialysis membranes: Effects of chronic complement activation. Kidney InternationaI, $25(1), 194-200$.

HCFA Release 1988 ESRD treatment center statistics. (1990). Dialysis and Transplantation, 19(1), 10 . Hoenich, N. A., Johnston, S. A. D., \& Buckley, P. (1984). Hemodialyzer reuse: Impact on function and biocompatibility. International Journal of Artificial Organs, $\underline{6}, 261$. 
Hopkins, P. E. (1982). On dialyzer reuse. Nephrology Nurse, $\underline{3}(2), 37$.

Kant, K. S., Pollack, V. E., \& Goetz, D. (1981). Multiple use of dialyzers: Safety and efficacy. Kidney International, 19(5), 729-738.

Lancaster, I. E. (1984). The patient with end stage renal disease, New York, NY: Wiley \& Sons.

LoBiondo-Wood, G., Haber, J. (1986). Nursing research. St. Louis, MO: C. V. Mosby.

Marriner, A. (1986). Nursing theorists and their work. St. Louis, MO: C. V. Mosby.

National Kidney Foundation. (1984). Revised standards for reuse of hemodialyzers. American Journal of Kidney Diseases, $\underline{3}(6), 466-469$.

Ogden, D. (1986). Clinical responses to new and reprocessed dialyzers. Dialysis and Transplantation, $15(6), 366-375$.

Papper, S. (1971). Elinical nephrology. Boston, MA: Little, Brown \& Co.

Recommended Practice for Reuse of Hemodialyzers. (1990). Arlington, VA: Association for the Advancement of Medical Instrumentation.

Report of national workshop on reuse of consumables in hemodialysis. (1984). Kidney Disease Coalition. Washington, D.C. 
Richards, C. J. (1986) . Comprehensive nephrology nursing. Boston, MA: Little, Brown \& Co. Senator, clinicians clash over safety of dialyzer reuse. (1987). Hospital Infection Control, 14(2), 17-32. Shott, S. (1990). Statistics for health professionals. Philadelphia, PA: W. B. Saunders Co. Simesen, A. W. (1985). Dialyzer reuse: A system for holding reliability to cost effectiveness. Dialysis and Iransplantation, $9(5), 933-936$.

Vanholder, A., Piron, M., Cubber, A., Vermaercke, N., \& Ringuir, S. (1987). Two year experience with automated reuse of hemodialyzers. Dialysis and Transplantation, $16(3), 132-138$.

Vlchek, D. (1987). Vlchek asks that we look at the facts of reuse. Nephrology News and Issues, 1(1), 32 . 
APPENDIX A

\section{Informed Consent}


AGREEMENT TO PARTICIPATE IN RESEARCH

SAN JOSE STATE UNIVERSITY

\author{
RESPONSIBLE INVESTIGATOR: \\ NANCY A. SMITH, R.N., B.S.N., C.N.N. \\ TITLE OE PROTOCOL: \\ A RETROSPECTIVE STUDY TO DETERMINE THE \\ RELATIONSHIP BETWEEN SELECTED HEMOCOMPONENTS, \\ ULTRAFIITRATION, AND BODY TEMPERATURE.
}

I have been asked to participate in a research study that is investigating the effects of dialyzer reuse on the

laboratory values of blood urea nitrogen, creatinine, ultrafiltration, and body temperature. The results of this study should further our understanding of dialyzer reuse.

I understand that:

1. I will be asked to continue dialyzer reuse for a period of six months, labs will continue to be drawn every other week as ordered by my physician.

2. The possible disadvantages have been explained to me previously, and include: the development of an antibody to red blood cells if formaldehyde rinsing is incomplete. However, when these antibodies have been observed, they do not seem to cause illness or effect kidney transplant success. Accidental dialysis on another person's dialyzer. long term exposure to residual amounts of formaldehyde. The long term effects are not known, but in many long terms studies no specific problems have been seen. The level of exposure to formaldehyde in the reused dialyzer is almost identical to the level of formaldehyde always circulating in the blood of all persons and therefore no additional load of formaldehyde is created by reuse when performed to the standards of the Department of Health Services. An increased possibility of developing a fever reaction or infection if reuse is not performed properly.

3. The possible benefits of this study to me are the establishment of a specified number of dialyzer reuses rather than the standard method of use until $20 \%$ of the fibers are clotted. This study will determine if there are increases in blood urea nitrogen, creatinine, body temperature, and a decrease in the ultrafiltration capacity of the dialyzer with reuse.

4. There are no alternative procedures associated with this study. 
5. The results of this study may be published, but any information that can be identified with me will remain confidential and will be disclosed only with my permission or as required by law.

6. Any questions about my participation in this study will be answered by Nancy A. Smith, R.N., (408) 758-6222. Complaints about the procedures may be presented to $\mathrm{Dr}$. Bobbye Gorenberg; Department Chair for faculty. For questions or complaints about research subject's rights, or in the event of research-related injury, contact serena Stanford, Ph.D. (Associate Academic Vice Presicient for Graduate Studies and Research) at (408) 924-2484.

7. My consent is given voluntarily without being coerced: I may refuse to participate in any part of this study, and may withdraw my consent at any time, without prejudice to my relations with San Jose State University or

8. I further understand that I am already participating in reuse and this study will involve analyzing data already gathered. 9. I have received a copy of this consent form for my
files.

I HAVE MADE A DECISION WHETHER OR NOT TO PARTICIPATE. MY SIGNATURE INDICATES THAT I HAVE READ THE INFORMATION PROVIDED ABOVE AND THAT I HAVE DECIDED TO PARTICIPATE.

DATE

SUBJECT'S SIGNATURE

INVESTIGATOR'S SIGNATURE 